Original article

\title{
Childhood Abuse and Early Menarche Among Peruvian Women
}

\author{
Yasmin V. Barrios, M.P.H. ${ }^{\mathrm{a}, *}$, Sixto E. Sanchez, M.D., M.P.H. ${ }^{\mathrm{b}, \mathrm{c}}$, \\ Christina Nicolaidis, M.D., M.P.H. ${ }^{\mathrm{d}}$, Pedro J. Garcia, M.D., M.P.H. ${ }^{\text {e, }}$, \\ Bizu Gelaye, Ph.D., M.P.H. ${ }^{\text {a }}$, Qiuyue Zhong, M.Sc. ${ }^{\text {, }}$, and Michelle A. Williams, Sc.D. ${ }^{a}$ \\ ${ }^{a}$ Department of Epidemiology, Harvard School of Public Health, Boston, Massachusetts \\ ${ }^{\mathrm{b}}$ Universidad Peruana de Ciencias Aplicadas, Lima, Peru \\ ${ }^{\mathrm{c}}$ Asociación Civil PROESA, Lima, Peru \\ d School of Social Work, Portland State University, Portland, Oregon \\ e Instituto Nacional Materno Perinatal, Lima, Peru
}

Article history: Received May 29, 2014; Accepted October 9, 2014

Keywords: Childhood abuse; Age at menarche; Peru

\section{A B S T R A C T}

Purpose: Childhood abuse has been associated with age of menarche in some studies, but not all, and few have assessed the independent associations of sexual and physical abuse with early menarche. We examined the association between childhood abuse and early menarche among pregnant women in Lima, Peru.

Methods: Multinomial logistic regression procedures were used to estimate odds ratios (OR) and $95 \%$ confidence intervals (CIs) for early menarche ( $\leq 11$ years) in relation to any physical or sexual childhood abuse, physical abuse only, sexual abuse only, and both physical and sexual abuse in a cohort of 1,499 pregnant (first trimester) women.

Results: Approximately 69\% of participants reported experiencing physical or sexual abuse in childhood. The frequencies of physical abuse only, sexual abuse only, and both physical and sexual abuse were $37.4 \%, 7.7 \%$, and $24.5 \%$, respectively. Compared with women who reported no childhood abuse, those who reported any childhood abuse had a 1.38 -fold increased odds of early menarche (95\% CI, 1.01-1.87). Compared with no abuse, the odds of early menarche was 1.60 -fold among women with childhood sexual abuse only (OR, 1.60; 95\% CI, .93-2.74) and 1.56-fold for those with both physical and sexual abuse (OR, 1.56; 95\% CI, 1.07-2.25) during childhood. Isolated physical abuse was weakly associated with early menarche (OR, 1.23; 95\% CI, .87-1.74). There was no clear evidence of association of childhood abuse with late menarche ( $\geq 15$ years).

Conclusions: Childhood abuse, particularly joint physical and sexual abuse, is associated with early menarche. Our findings add to an expanding body of studies documenting the enduring adverse health consequences of childhood abuse.

(c) 2015 Society for Adolescent Health and Medicine. All rights reserved.

\section{IMPLICATIONS AND CONTRIBUTION}

Childhood abuse is prevalent among pregnant women in Peru, a population known to have a high burden of gender-based violence across the life course. This study documents the association of childhood abuse with early menarche among lowincome Peruvian women. Health care providers should screen women for the past and current abuse.

\footnotetext{
Conflicts of Interest: The authors have no conflicts of interest to declare. Disclaimer: The National Institutes of Health had no further role in study design; in the collection, analysis, and interpretation of data; in the writing of the report; and in the decision to submit the article for publication.

* Address correspondence to: Yasmin V. Barrios, M.P.H., Department of Epidemiology, Harvard School of Public Health, 677 Huntington Avenue, K502A, Boston, MA 02115.

E-mail address: ybarrios@hsph.harvard.edu (Y.V. Barrios).
}

Menarche is an important milestone of sexual development for a woman. Age at menarche signals the end of puberty and the beginning of her reproductive life [1,2]. According to Belsky, Steinberg, and Draper's evolutionary theory of socialization and development, children in highly stressful family context where resources are limited, parents are harsh, rejecting, insensitive, or inconsistent (possibly physiologically or physically abusive) 
develop psychological and behavioral issues and experience early maturation and puberty. They also engage in earlier sexual activity and have short unstable pair bonds with little parental investment in their own offspring $[3,4]$.

Reaching menarche at an early age has been associated with a broad spectrum of behavioral, reproductive, and health outcomes including early age at initiation of sexual activity [5-7] and first pregnancy [6,8], diminished ovarian function [9], increased risks of obesity [10], autoimmune disorders [11], psychiatric disorders [12], metabolic syndrome, type 2 diabetes, cardiovascular disorders $[1,13,14]$, medical complications of pregnancy including preeclampsia [15,16], and gestational diabetes [10].

Consistent with Belsky, Steinberg, and Draper's theory, an emerging literature implicates childhood physical and sexual abuse, a specific type of early life stressor, as a risk factor for reaching menarche early [17-19]. For instance, Romans et al. [18] using the Otago Women's Health Child Sexual Abuse Survey noted that a number of adverse childhood experiences including childhood physical and sexual abuse preceded early menarche. In concurrence, Henrichs et al. [20] in a nationally representative sample found increased odds of early menarche associated with childhood sexual abuse. Similar results were reported by other investigators [8]. However, few investigators have assessed the relationship between child sexual abuse and age at menarche among Latin American women. Using information from a large pregnancy cohort, we conducted the present cross-sectional analysis to assess the extent to which, if at all, women's early experience with physical and/or sexual abuse is associated with early age at menarche. We sought to explore the independent and joint association of physical and sexual abuse with risk of early age at menarche among low-income Peruvian women, a population known to be exposed to a high prevalence of genderbased violence across the life course, with up to $40 \%$ intimate partner violence and $67 \%$ childhood physical punishment [21-24]. Documentation of associations of childhood abuse with early age at menarche in this population may have important clinical implication insofar as alerting health care providers to the need to evaluate and screen women for the past and current abuse.

\section{Methods}

\section{The Pregnancy Outcomes, Maternal and Infant Study study}

The population for the present study was drawn from participants of the ongoing Pregnancy Outcomes, Maternal and Infant Study (PrOMIS) cohort, designed to examine maternal social and behavioral risk factors of preterm birth and other adverse pregnancy outcomes. The study population consists of women attending prenatal care clinics at the Instituto Nacional Materno Perinatal (INMP) in Lima, Peru. The INMP is the primary reference establishment for maternal and perinatal care operated by the Ministry of Health of the Peruvian government. Recruitment began in February 2012. Women eligible for inclusion were those who initiated prenatal care prior to 16 weeks gestation. Women were ineligible if they were aged younger than 18 years, did not speak and read Spanish, or had completed more than 16 weeks of gestation.

Enrolled participants were invited to take part in an interview in which trained research personnel used a structured questionnaire to elicit information regarding maternal sociodemographics, lifestyle characteristics, medical and reproductive histories, and early life experiences of abuse. All participants provided written informed consent. The institutional review boards of the INMP, Lima, Peru, and the Harvard School of Public Health Office of Human Research Administration, Boston, MA, approved all procedures used in this study.

\section{Analytical population}

The study population for this report is derived from information collected from those participants who enrolled in the PrOMIS cohort between February 2012 and March 2013. During this period, 1,810 eligible women were approached, and 1,556 (86\%) agreed to participate. Fifty-seven were excluded from the present analysis because of missing information concerning their experience with abuse in childhood and/or missing information for age at menarche. Women excluded from this analysis did not differ in regards to sociodemographic and lifestyle characteristics as compared with those included. As shown in Table 1, a total of 1,499 women remained for analysis. Participants' age was categorized as follows: $18-20,21-29,30-34$, and $\geq 35$ years. Other sociodemographic variables were categorized as follows: maternal ethnicity (Mestizo vs. others); educational attainment ( $\leq 6,7-12$, and $>12$ completed years of schooling); marital status (married and living with partner vs. others); employment status (employed vs. not employed); access to basic foods (very hard/ hard, somewhat hard, and not very hard); parity (nulliparous vs. multiparous); planned pregnancy (yes vs. no); self-reported health in the past year (good vs. poor); and gestational age at

\section{Table 1}

Sociodemographic and reproductive characteristics of the study population by types of childhood abuse in Lima, Peru $(\mathrm{N}=1,499)$

\begin{tabular}{|c|c|c|c|c|c|c|c|c|}
\hline \multirow[t]{2}{*}{ Characteristic } & \multicolumn{2}{|c|}{$\begin{array}{l}\text { No abuse } \\
(\mathrm{N}=456)\end{array}$} & \multicolumn{2}{|c|}{$\begin{array}{l}\text { Physical } \\
\text { abuse only } \\
(\mathrm{N}=561)\end{array}$} & \multicolumn{2}{|c|}{$\begin{array}{l}\text { Sexual } \\
\text { abuse only } \\
(\mathrm{N}=116)\end{array}$} & \multicolumn{2}{|c|}{$\begin{array}{l}\text { Physical and } \\
\text { sexual abuse } \\
(\mathrm{N}=366)\end{array}$} \\
\hline & $\mathrm{n}$ & $\%$ & $\mathrm{n}$ & $\%$ & $\mathrm{n}$ & $\%$ & $\mathrm{n}$ & $\%$ \\
\hline Age (years) ${ }^{a}$ & \multicolumn{2}{|c|}{$27.4 \pm 6.2$} & \multicolumn{2}{|c|}{$28.0 \pm 6.2$} & \multicolumn{2}{|c|}{$27.9 \pm 6.3$} & \multicolumn{2}{|c|}{$28.7 \pm 6.2$} \\
\hline \multicolumn{9}{|l|}{ Age (years) } \\
\hline $18-20$ & 28 & 6.1 & 30 & 5.4 & 9 & 7.8 & 19 & 5.2 \\
\hline $21-29$ & 285 & 62.5 & 318 & 56.7 & 66 & 56.9 & 188 & 51.4 \\
\hline $30-34$ & 76 & 16.7 & 116 & 20.7 & 18 & 15.5 & 87 & 23.8 \\
\hline$\geq 35$ & 67 & 14.7 & 97 & 17.3 & 23 & 19.8 & 72 & 19.7 \\
\hline \multicolumn{9}{|l|}{ Education (years) } \\
\hline$\leq 6$ & 19 & 4.2 & 24 & 4.3 & 2 & 1.7 & 19 & 5.2 \\
\hline$\overline{7}-12$ & 260 & 57.0 & 308 & 54.9 & 62 & 53.5 & 211 & 57.7 \\
\hline$>12$ & 176 & 38.6 & 228 & 40.6 & 50 & 43.1 & 135 & 36.9 \\
\hline Mestizo & 351 & 77.0 & 425 & 75.8 & 90 & 77.6 & 266 & 72.7 \\
\hline $\begin{array}{l}\text { Married/living with } \\
\text { a partner }\end{array}$ & 375 & 82.2 & 458 & 81.6 & 90 & 77.6 & 297 & 81.2 \\
\hline Nulliparous & 256 & 56.1 & 274 & 48.8 & 62 & 53.5 & 161 & 44.0 \\
\hline Employed & 195 & 42.8 & 243 & 43.3 & 53 & 45.7 & 157 & 42.9 \\
\hline \multicolumn{9}{|c|}{ Access to basic foods } \\
\hline Very hard/hard & 65 & 14.3 & 109 & 19.4 & 25 & 21.6 & 80 & 21.9 \\
\hline Somewhat hard & 132 & 29.0 & 169 & 30.1 & 42 & 36.2 & 143 & 39.1 \\
\hline Not very hard & 259 & 56.8 & 282 & 50.3 & 49 & 42.2 & 143 & 39.1 \\
\hline Planned pregnancy & 202 & 44.3 & 249 & 44.4 & 44 & 37.9 & 137 & 37.4 \\
\hline \multicolumn{9}{|c|}{$\begin{array}{l}\text { Self-reported health } \\
\text { status in the } \\
\text { past year }\end{array}$} \\
\hline Good & 346 & 75.9 & 392 & 69.9 & 75 & 64.7 & 214 & 58.5 \\
\hline Poor & 102 & 22.4 & 162 & 28.9 & 39 & 33.6 & 144 & 39.3 \\
\hline $\begin{array}{l}\text { Gestational age at } \\
\text { interview }^{\mathrm{a}}\end{array}$ & \multicolumn{2}{|c|}{$9.6 \pm 3.4$} & \multicolumn{2}{|c|}{$9.9 \pm 3.4$} & \multicolumn{2}{|c|}{$9.5 \pm 3.1$} & \multicolumn{2}{|c|}{$9.9 \pm 3.4$} \\
\hline
\end{tabular}

Because of missing data, percentages may not add up to $100 \%$.

${ }^{\text {a }}$ Mean \pm standard deviation. 
interview. Self-reported age at menarche, categorized into three groups ( $\leq 11,12-14$, and $\geq 15$ years), was based on cut points reported by previous investigators $[10,15,20]$.

\section{Childhood abuse assessment}

We used the Childhood Physical and Sexual Abuse Questionnaire to elicit information concerning participants' experiences with physical and sexual abuse in childhood [25]. The instrument consists of eight questions concerning abuse taken from the Centers for Disease Control and Prevention Adverse Childhood Experiences Study. Respondents were rated as having experienced childhood abuse if, before the age of 18 years, they reported that an older person touched them, they were made to touch someone else in a sexual way, or intercourse was attempted or completed (sexual abuse) or that they were hit, kicked, or beaten often and/or their life was seriously threatened (physical abuse). Participants who responded "no" to all questions regarding sexual and physical abuse were categorized as "no abuse." Those responding "yes" to only physical abuse questions were categorized as "both physical abuse only" and those responding "yes" to only sexual abuse questions were categorized as "sexual abuse only." Those responding "yes" to any physical abuse questions and "yes" to any sexual abuse questions were categorized as having experienced "physical and sexual abuse." Participants who responded "yes" to any questions of physical abuse or "yes" to any questions of sexual abuse or yes to both abuse types were categorized as having experienced "any abuse."

\section{Statistical analyses}

Frequency distributions of maternal sociodemographic characteristics by types of history of childhood abuse were examined. Multinomial logistic regression procedures were used to calculate maximum likelihood estimates of odds ratios (ORs) and 95\% confidence intervals (CIs) for early age and late age at menarche in relation to childhood abuse (any abuse, physical only, sexual only, and both physical and sexual) while adjusting for potential confounders. Those with "no abuse" and "menarche age (12-14 years)" served as the reference group. Potential confounders were selected a priori on the basis of their hypothesized relationship between childhood abuse and age at menarche. The variables included maternal age, education, employment status, parity, and difficulty paying for basic foods $[15,19,21,26,27]$. All statistical analyses were performed using SAS 9.3 (SAS Institute, Cary, NC). All reported $p$ values are two tailed with statistical significance set at .05 .

\section{Results}

Approximately $69.6 \%$ of participants reported having experienced any physical or sexual abuse during their childhood. The frequencies of physical abuse only, sexual abuse only, and both physical and sexual abuse were $37.4 \%, 7.7 \%$, and $24.5 \%$, respectively. Overall, the mean age of menarche among participants was 12.9 years (standard deviation [SD], 1.6 years).

Sociodemographic and lifestyle characteristics of study participants according to history and type of childhood abuse are shown in Table 1 . The mean age of participants was 28.0 years $(\mathrm{SD}, \pm 6.2)$ and approximately $81 \%$ were married or were living with their partner. Approximately 57\% reported being unemployed, about 55\% completed between 7 and 12 years of education and 40\% completed more than 12 years. Among the nonabused women, $14.3 \%$ reported that it was very hard to access basic foods and this proportion was higher for women who reported experiencing sexual abuse only or both sexual and physical abuse in childhood (21.6\% and $21.9 \%$, respectively). Approximately $75 \%$ of the nonabused women self-reported their health status in the past year as having been good, whereas only $58 \%$ of those who had a history of both physical and sexual abuse reported their health status as good.

Approximately $18.3 \%$ of participants were classified as having had early age at menarche ( $\leq 11$ years), and $16.8 \%$ of participants were classified as having had a late age at menarche ( $\geq 15$ years). For this analysis, the remaining $64.8 \%$ women with an age at menarche between 12 and 14 years and those who experienced no abuse comprised the referent group. As shown in Table 2, a history of any childhood physical or sexual abuse was associated with a 1.40-fold increased odds of early menarche (OR, 1.40; 95\% CI, 1.03-1.90). The association remained but was slightly attenuated after adjustment for age, educational attainment, employment status, parity, and difficulty paying for basics (adjusted odds ratio [aOR], 1.38; 95\% CI, 1.01-1.87). There was no clear evidence of an association between childhood physical or sexual abuse and odds of late age at menarche (aOR, 1.16;95\% CI, .85-1.58). We next evaluated the association of early and late age at menarche with each specific type of childhood abuse. As shown in the bottom panel of Table 2, compared with women who experienced no abuse during childhood, the odds of early menarche was 1.60 -fold for those who reported experiencing sexual abuse only (aOR, 1.60; 95\% CI, .93-2.74) and 1.56-fold among those who reported experiencing both physical and sexual abuse (aOR, 1.56; 95\% CI, 1.07-2.25) during childhood, although statistical significance was not achieved for sexual abuse only. There was no clear evidence of an association of isolated physical abuse during childhood and risk of early age at menarche (aOR, 1.23; 95\% CI, .87-1.74). Those with isolated experience of sexual abuse in childhood were 1.65 times as likely to have a late age at menarche compared with women who experienced no abuse during childhood; however, the association did not reach statistical significance after we adjusted for confounders (aOR, 1.65; 95\% CI, .97-2.81).

\section{Discussion}

We found that a history of any childhood physical or sexual abuse was associated with 1.40-fold increased odds of reporting early age at menarche among pregnant Peruvian women. Additionally, we found that a history of both physical and sexual childhood abuse was associated with a 1.56-fold increased odds of early menarche. Our results are consistent with previous studies which investigated both physical and sexual abuse and found associations between each type of abuse and early menarche $[8,18,20,26-28]$. Our results are also consistent with previous studies that focused solely on childhood sexual abuse and which found a positive association between sexual abuse and early menarche $[17,19,29,30]$.

Prior studies regarding age at menarche rely on retrospective reporting. For example, Romans et al. [18] conducted a mailed survey of 350 women to investigate a number of childhood factors thought to be associated with early menarche. They found that women with early menarche were more likely to report a history of childhood sexual abuse and severe physical punishment. They further analyzed those women who reported sexual abuse and found that the association with early menarche was 
Table 2

Association between childhood abuse and timing of menarche $(\mathrm{N}=1,499)^{\mathrm{a}}$

\begin{tabular}{|c|c|c|c|c|c|c|c|}
\hline \multirow[t]{2}{*}{ Childhood abuse } & \multirow{2}{*}{$\begin{array}{l}\text { Menarche age } \\
(12-14 \text { years); } \\
\mathrm{N}=972 \\
\mathrm{n}(\%)\end{array}$} & \multicolumn{3}{|c|}{ Early menarche ( $\leq 11$ years); $N=275$} & \multicolumn{3}{|c|}{ Late menarche ( $\geq 15$ years); $N=252$} \\
\hline & & $\mathrm{n}(\%)$ & $\begin{array}{l}\text { Unadjusted } \\
\text { OR }(95 \% \mathrm{CI})\end{array}$ & $\begin{array}{l}\text { Adjusted } \\
\text { OR }(95 \% \mathrm{CI})^{\mathrm{b}}\end{array}$ & $\mathrm{n}(\%)$ & $\begin{array}{l}\text { Unadjusted } \\
\text { OR }(95 \% \mathrm{CI})\end{array}$ & $\begin{array}{l}\text { Adjusted } \\
\text { OR }(95 \% \mathrm{CI})^{\mathrm{b}}\end{array}$ \\
\hline No abuse & $314(32.3)$ & $70(25.5)$ & Reference & Reference & $72(28.6)$ & Reference & Reference \\
\hline Any abuse & $658(67.7)$ & 205 (74.6) & $1.40(1.03-1.90)$ & $1.38(1.01-1.87)$ & $180(71.4)$ & $1.20(.88-1.62)$ & $1.16(.85-1.58)$ \\
\hline \multicolumn{8}{|l|}{ Types of abuse } \\
\hline No abuse & $314(32.3)$ & $70(25.5)$ & Reference & Reference & $72(28.6)$ & Reference & Reference \\
\hline Physical abuse only & $362(37.2)$ & $100(36.4)$ & $1.24(.88-1.75)$ & $1.23(.87-1.74)$ & 99 (39.3) & $1.20(.85-1.68)$ & $1.17(.83-1.64)$ \\
\hline Sexual abuse only & $66(6.8)$ & $24(8.7)$ & $1.63(.96-2.78)$ & $1.60(.93-2.74)$ & $26(10.3)$ & $1.72(1.02-2.89)$ & $1.65(.97-2.81)$ \\
\hline Physical and sexual abuse & $230(23.7)$ & $81(29.5)$ & $1.58(1.10-2.27)$ & $1.56(1.07-2.25)$ & $55(21.8)$ & $1.04(.71-1.54)$ & $1.01(.68-1.50)$ \\
\hline
\end{tabular}

Bold values are statistically significant values.

$\mathrm{CI}=$ confidence interval; $\mathrm{OR}=$ odds ratio.

a Multinomial logistic regression model was built where those with "No abuse" and "Menarche age (12-14 years)" served as the reference group.

b Adjusted for age (years), education (years), employment status (yes vs. no), parity (nulliparous vs. multiparous), and difficulty paying for the very basics (very hard or hard, somewhat hard, and not very hard).

strongest if the sexual abuse involved attempted or completed intercourse [18]. A second study of a nationally representative sample of women in the United States evaluated 11 childhood adversities and found that physical abuse, sexual abuse, family violence, and parental mental illness were associated with early menarche [20]. After adjusting for co-occurring adversities that happened before menarche, only the relationship between childhood sexual abuse and early menarche remained (OR, 1.77; 95\% CI, 1.21-2.60) [20]. Brown et al. [8] also examined the sexual trajectories of abused and neglected youth during a prospective study with the Children in the Community Study of New York. Their results showed that among those children experiencing physical abuse, the mean age at menarche was 12.3 years and for those who experienced two or more episodes of sexual abuse, it was 11.8 years, with the comparison group having a mean age of 12.4 years [8].

Retrospective reports from women participating in the Nurses' Health Study II showed that 57\% had experienced some form of physical or sexual abuse (36\% physical abuse only, $8 \%$ sexual abuse only, and $13 \%$ both physical and sexual abuse) during childhood, and the overall median age at menarche was 12 years [27]. Their results showed that, compared with women who reported no childhood abuse, those with a history of severe physical abuse (aOR, 1.22; 95\% CI, 1.10-1.37) and sexual abuse (aOR, 1.20; 95\% CI, 1.10-1.37) had a positive association with early menarche ( $<11$ years) [27]. They also reported finding a doseresponse association between severity of physical abuse and delayed onset of menarche ( $>15$ years) but found no such association between sexual abuse and late menarche [27]. In our analysis of late menarche, we did not find a statistically significant association with a history of childhood physical or sexual abuse. The analysis of abuse and age at menarche among the Black Women's Health Study participants considered independent and joint experience of abuse types [26]. Their results showed an adjusted relative risk (RR) for early menarche of 1.27 (95\% CI, 1.20-1.35) for those having experienced sexual abuse only and a weak, but statistically significant, RR of 1.05 (95\% CI, 1.01-1.09) for physical abuse only and RR of 1.22 (95\% CI, $1.16-1.28$ ) for both physical and sexual abuse relative to no abuse [26]. They suggested that self-reported history of sexual abuse, which was confirmed to have occurred before menarche, increased risk of early menarche ( $<12$ years) and that the association between childhood physical abuse was unclear [26].
Specifically considering sexual abuse, the findings of our analysis provide support for an association between childhood abuse and early age at menarche but cannot speak to the temporal association between the two. The more traditional hypothesis is that there is greater vulnerability and increased desirability to perpetrators among girls developing early [26]. Zabin et al. [29] set out to test that hypothesis along with a second; which considers that abuse occurs before puberty and menarche specifically because the perpetrator finds it appealing that the victim is a child and has not yet developed adult female traits. Their results showed that, although abuse did occur during puberty while secondary sex features were developing, the abuse generally began before the onset of puberty; with $63 \%$ of the cases reporting that their abuse began before 3 years or more before menarche and greater than $18 \%$ of the cases reporting their abuse occurred 8 years before menarche, with an average age of 9 years at the onset of abuse [29]. It was a small study of 323 African-American and multiethnic Caucasian women in the United States, but they were able to show that many sexual abuse events occurred long before puberty. In a second effort to investigate the direction of the relationship between menarche and childhood sexual abuse, Kendall et al. [17] interviewed 365 adults and found that the average age of onset of sexual abuse was 7 years, indicating that abuse preceded the onset of puberty. A short survey of childhood abuse among children admitted to a referral hospital in Lima, Peru, was able to determine that among the admitted sexually abused girls, the average age of reported abuse was 9 years [23]. Collectively, the U.S. studies help to clarify the possible temporal relation between abuse experience and early menarche. Moreover, these studies underscore the importance of future investigations among diverse populations designed to assess biopsychosocial mechanisms whereby childhood physical and sexual abuse may contribute to accelerated sexual maturation [29].

Pubertal development is primarily controlled through the hypothalamic-pituitary-axis (HPA) and the hypothalamicpituitary-gonadal (HPG) axis, indicating that a biological plausible association between childhood abuse and early age at menarche may exist because of the biological effect that severe stress or childhood trauma has on the HPA and HPG axis [26]. However, further research is required to understand the full interaction between HPA and HPG to explain the biological underpinnings of the associations found between childhood abuse and early age at menarche. Other risk factors associated with 
early menarche include a diverse set of psychosocial, behavioral, environmental, and genetic factors [3,31-33]. Notably, birth size [31] and early life obesity [34], physical inactivity [35], and calorie dense diets [36] have been implicated as important determinants of early menarche.

Available evidence also indicates that age at menarche is decreasing rapidly for virtually all populations [37,38]. Reasons for the downward trend are not fully understood, but it is likely that nongenetic factors are important in these pronounced secular trends. Yet, nationally representative data for developing countries regarding age at menarche are lacking and make it difficult to make comparisons between and within countries. Parent et al. [39] conducted a review of global variations of age at menarche and found only a small number of studies from Latin American countries. For instance, mean or median age at menarche was reported for Guatemalan (13.8 years), Columbian (13.4 years), Chilean (12.5 years), and Venezuelan (12.6 years) girls [39]. In the United States, population-based studies that have assessed racial differences in age at menarche demonstrated that non-Hispanic black women reported the earliest median age (12.1 years), non-Hispanic white women reported the latest (12.6 years), and Mexican-American women fell inbetween (12.3 years) [40]. Studies measuring differences in age of menarche between racial/ethnic groups are limited and few explore childhood abuse on menarche in developing countries with a high prevalence of childhood abuse, such as Peru [21-23], in part because of the lack of consensus on how to define harsh physical discipline that is widespread and culturally acceptable [22]. This study is the first, to our knowledge, to examine the relationship between childhood physical and sexual abuse and age at menarche among a Peruvian cohort.

Our relatively large sample size allowed us to control for several potential confounding factors and we had the opportunity to examine the relations according to type of childhood abuse experienced. However, several limitations should be acknowledged. First, we did not have detailed information about the specific timing and severity of childhood abuse in this crosssectional study and we cannot determine a temporal relation. Second, age at menarche and experience with childhood abuse were assessed using participant self-report and the other covariates were related to current status of the women. Hence, because of self-report, these data are subject to misclassification or recall bias. Also, this analysis was conducted among currently pregnant women and limits generalizability. Finally, although we used multivariable logistic procedures to adjust for confounding, we cannot rule out the possibility of some residual confounding by unmeasured factors. Specifically, we neither had information regarding nutrition, physical activity, or weight at the time of menarche nor knew the mother's age of the first menstruation.

Further research on the types, severity, duration, and perpetrators of childhood abuse is needed, as well as research aimed at understanding the biological mechanisms through which childhood abuse is associated with age at menarche. Early menarche may be a signal to health care providers or supportive caregivers that the young girl may be suffering from abuse. Identifying young girls who are suffering from abuse or experiencing early menarche and providing them support during this potentially difficult time may help mitigate negative psychosocial and health outcomes. Greater efforts are needed to prevent child abuse and its many health consequences.

A history of childhood abuse is prevalent among pregnant Peruvian women. Any physical or sexual childhood abuse is associated with early menarche. Regarding abuse types, we found that experiencing childhood physical and sexual abuse combined and isolated sexual abuse increased the odds of early menarche. Our findings add to an expanding body of literature documenting the enduring adverse health implications of childhood abuse.

\section{Acknowledgments}

The authors wish to thank the dedicated staff members of Asociacion Civil Proyectos en Salud (PROESA), Peru, and Instituto Materno Perinatal, Peru, for their expert technical assistance with this research.

\section{Funding Sources}

This research was supported by an award from the National Institutes of Health, the Eunice Kennedy Shriver Institute of Child Health and Human Development (R01-HD-059835).

\section{Supplementary Data}

Supplementary data related to this article can be found at http://dx.doi.org/10.1016/j.jadohealth.2014.10.002.

\section{References}

[1] Lakshman R, Forouhi NG, Sharp SJ, et al. Early age at menarche associated with cardiovascular disease and mortality. J Clin Endocrinol Metab 2009; 94:4953-60.

[2] Chavarro J, Villamor E, Narvaez J, Hoyos A. Socio-demographic predictors of age at menarche in a group of Colombian university women. Ann Hum Biol 2004:31:245-57.

[3] Wierson M, Long PJ, Forehand RL. Toward a new understanding of early menarche: The role of environmental stress in pubertal timing. Adolescence 1993;28:913-24.

[4] Belsky J, Steinberg L, Draper P. Childhood experience, interpersonal development, and reproductive strategy: And evolutionary theory of socialization. Child Dev 1991;62:647-70.

[5] Zabin LS, Smith EA, Hirsch MB, Hardy JB. Ages of physical maturation and first intercourse in black teenage males and females. Demography 1986; 23:595-605.

[6] Flannery DJ, Rowe DC, Gulley BL. Impact of pubertal status, timing, and age on adolescent sexual experience and delinquency. J Adolesc Res 1993;8: $21-40$.

[7] Moore SR, Harden KP, Mendle J. Pubertal timing and adolescent sexual behavior in girls. Dev Psychol 2014;50:1734-45.

[8] Brown J, Cohen P, Chen H, et al. Sexual trajectories of abused and neglected youths. J Dev Behav Pediatr 2004;25:77-82.

[9] Weghofer A, Kim A, Barad DH, Gleicher N. Age at menarche: A predictor of diminished ovarian function? Fertil Steril 2013;100:1039-43.

[10] Dishi M, Enquobahrie DA, Abetew DF, et al. Age at menarche, menstrual cycle characteristics and risk of gestational diabetes. Diabetes Res Clin Pract 2011;93:437-42.

[11] Karlson EW, Mandl LA, Hankinson SE, Grodstein F. Do breast-feeding and other reproductive factors influence future risk of rheumatoid arthritis? Results from the Nurses' Health Study. Arthritis Rheum 2004;50:3458-67.

[12] Natsuaki MN, Leve LD, Mendle J. Going through the rites of passage: Timing and transition of menarche, childhood sexual abuse, and anxiety symptoms in girls. J Youth Adolesc 2011;40:1357-70.

[13] Solomon CG, Hu FB, Dunaif A, et al. Menstrual cycle irregularity and risk for future cardiovascular disease. J Clin Endocrinol Metab 2002;87:2013-7.

[14] Chen L, Zhang C, Yeung E, et al. Age at menarche and metabolic markers for type 2 diabetes in premenopausal women: The BioCycle study. J Clin Endocrinol Metab 2011;96:E1007-12.

[15] Rudra CL, Williams MA. BMI as a modifying factor in the relations between age at menarche, menstrual cycle characteristics, and risk of preeclampsia. Gynecol Endocrinol 2005;21:200-5.

[16] Abetew DF, Enquobahrie DA, Dishi M, et al. Age at menarche, menstrual characteristics, and risk of preeclampsia. ISRN Obstet Gynecol 2011;2011: 472083.

[17] Kendall-Tackett KA, Simon AF. Molestation and the onset of puberty: Data from 365 adults molested as children. Child Abuse Negl 1988;12:73-81. 
[18] Romans SE, Martin JM, Gendall K, Herbison GP. Age of menarche: The role of some psychosocial factors. Psychol Med 2003;33:933-9.

[19] Vigil JM, Geary DC, Byrd-Craven J. A life history assessment of early childhood sexual abuse in women. Dev Psychol 2005;41:553-61.

[20] Henrichs KL, McCauley HL, Miller E, et al. Early menarche and childhood adversities in a nationally representative sample. Int J Pediatr Endocrinol 2014;2014:14.

[21] Cripe SM, Sanchez SE, Perales MT, et al. Association of intimate partner physical and sexual violence with unintended pregnancy among pregnant women in Peru. Int J Gynaecol Obstet 2008;100:104-8.

[22] Gage AJ, Silvestre EA. Maternal violence, victimization, and child physical punishment in Peru. Child Abuse Negl 2010;34:523-33.

[23] Escalante-Romero L, Huamani C, Serpa H, et al. Child and adolescent abuse recorded at a national referral hospital, 2006-2011. Rev Peru Med Exp Salud Publica 2012;29:28-34.

[24] Sanchez SE, Qiu C, Perales MT, et al. Intimate partner violence (IPV) and preeclampsia among Peruvian women. Eur J Obstet Gynecol Reprod Biol 2008; 137:50-5.

[25] Felitti VJ, Anda RF, Nordenberg D, et al. Relationship of childhood abuse and household dysfunction to many of the leading causes of death in adults. The Adverse Childhood Experiences (ACE) Study. Am J Prev Med 1998; 14:245-58.

[26] Wise LA, Palmer JR, Rothman EF, Rosenberg L. Childhood abuse and early menarche: Findings from the black women's health study. Am J Public Health 2009;99(Suppl 2):S460-6.

[27] Boynton-Jarrett R, Wright RJ, Putnam FW, et al. Childhood abuse and age at menarche. J Adolesc Health 2013;52:241-7.

[28] Boynton-Jarrett R, Harville EW. A prospective study of childhood social hardships and age at menarche. Ann Epidemiol 2012;22:731-7.
[29] Zabin LS, Emerson MR, Rowland DL Childhood sexual abuse and early menarche: The direction of their relationship and its implications. J Adolesc Health 2005;36:393-400.

[30] Grimstad H, Schei B. Pregnancy and delivery for women with a history of child sexual abuse. Child Abuse Negl 1999;23:81-90.

[31] Sorensen K, Juul A, Christensen K, et al. Birth size and age at menarche: A twin perspective. Hum Reprod 2013;28:2865-71.

[32] Tanikawa C, Okada Y, Takahashi A, et al. Genome wide association study of age at menarche in the Japanese population. PLoS One 2013;8:e63821.

[33] Allison CM, Hyde JS. Early menarche: Confluence of biological and contextual factors. Sex Roles 2013;68:55-64.

[34] Davison KK, Susman EJ, Birch LL. Percent body fat at age 5 predicts earlier pubertal development among girls at age 9. Pediatrics 2003;111:815-21.

[35] Moisan J, Meyer F, Gingras S. Leisure physical activity and age at menarche. Med Sci Sports Exerc 1991;23:1170-5.

[36] Berkey CS, Gardner JD, Frazier AL, Colditz GA. Relation of childhood diet and body size to menarche and adolescent growth in girls. Am J Epidemiol 2000; $152: 446-52$

[37] McDowell MA, Brody DJ, Hughes JP. Has age at menarche changed? Results from the National Health and Nutrition Examination Survey (NHANES) 1999-2004. J Adolesc Health 2007;40:227-31.

[38] Lehmann A, Scheffler C, Hermanussen M. The variation in age at menarche: An indicator of historic developmental tempo. Anthropol Anz 2010;68: 85-99.

[39] Parent AS, Teilmann G, Juul A, et al. The timing of normal puberty and the age limits of sexual precocity: Variations around the world, secular trends, and changes after migration. Endocr Rev 2003;24:668-93.

[40] Chumlea WC, Schubert CM, Roche AF, et al. Age at menarche and racial comparisons in US girls. Pediatrics 2003;111:110-3. 